\title{
Recent Results from BESIII
}

\author{
Frederick A. Harris, for the BESIII Collaboration \\ University of Hawaii, Honolulu, Hawaii 96822, USA \\ E-mail: fah@phys.hawaii.edu
}

The BEPCII collider operates in the tau-charm center of mass energy region from $2 \mathrm{GeV}$ to 4.6 $\mathrm{GeV}$ with a design luminosity of $1 \times 10^{33} \mathrm{~cm}^{-2} \mathrm{~s}^{-1}$. Samples of $106 \mathrm{M} \psi^{\prime}$ events and 225 $\mathrm{M} J / \psi$ events were recorded by the BESIII detector in the summer of 2009. Results based on these samples are described, including $h_{c}$ studies, measurements of $\chi_{c J}$ decays, confirmations of the BESII $X(1660)$ and $X(1835)$ resonances, $f_{0}(980)-a_{0}(980)$ mixing signals, and $\psi^{\prime} \rightarrow$ $\gamma P\left(P=\pi^{0}, \eta, \eta^{\prime}\right)$. Also described are the BESIII beam energy measurement system, which will be important for an improved tau mass measurement, and the future BESIII program.

XLIX International Winter Meeting on Nuclear Physics, BORMIO2011

January 24-28, 2011

Bormio, Italy 


\section{Introduction}

On November 11, 1974, Burt Richter (SLAC) announced the discovery of the $\psi$ particle [1] and Sam Ting (Brookhaven) announced the discovery of the $J$ [2], leading to the present day designation of $J / \psi$. This discovery was called the November Revolution, because the $J / \psi$ with the large mass of $3.096 \mathrm{GeV} / c^{2}$, but a very narrow width, was soon interpreted as being made up of a charm quark and an anti-charm quark. Such particles are said to have hidden charm and are called charmonium. In 1976, Richter and Ting received the Nobel prize for their discovery.

The existence of the charm quark, the fourth quark, had been predicted by Glashow, Iliopoulos, and Maiani to explain the absence of strangeness changing neutral currents [3]. Furthermore, its discovery finally convinced physicists of the reality of the quark model. Earlier, many considered the quark model a nice scheme to categorize the existing hadrons but were skeptical about their existence. The discovery of the fifth quark, the $B$ quark occurred a few years later.

The SLAC discovery took place at the SPEAR collider where electrons and positrons with equal (but opposite) momentum collided head on. In this scheme, all the energy of the electrons and positrons can be used to create new particles, like the $J / \psi$. As the energies of the electrons and positrons in SPEAR were slowly increased from lower energy, the $J / \psi$ showed up as a very large and narrow increase in the cross section of $e^{+}+e^{-} \rightarrow$ hadrons at about $3.1 \mathrm{GeV}$ in the center of mass energy. Rather surprisingly, not far from the $J / \psi$ is the threshold for the production of tau lepton pairs; the tau lepton is the third lepton in the series of electron, muon, and tau. The tau was discovered at SPEAR in a series of experiments between 1974 and 1976 by Martin Perl [4]. Perl received the Nobel Prize in 1995.

The center of mass energy region from about 3 to $5 \mathrm{GeV}$ is called the tau-charm region and contains the threshold for producing tau pairs, $J / \psi$ particles, and many cousins (also charmonium) of the $J / \psi$. If the center of mass energy is above $3.77 \mathrm{GeV}$, open charmed particles containing a single charm or anti-charm quark may be produced. The tau-charm region is extremely interesting with many hidden charm and open charm resonances being produced.

In the early 1990s, the Beijing Electron Positron Collider (BEPC) began operation. The collider and the detector, the Beijing Spectrometer (BES), were built with encouragement and advice from SLAC. BES operated until 1994 when it was upgraded to BESII. BESII stopped operation in 2004, when it was replaced by a new detector, BESIII, and BEPC was upgraded to a two ring collider, BEPCII.

During 2009, BESIII acquired samples of $106 \mathrm{M} \psi^{\prime}$ events, or four times the CLEOc sample, and $226 \mathrm{M} J / \psi$ events, or about four times the BESII $J / \psi$ sample. Results in this paper are based on these data sets, which are the world's largest.

\section{BEPCII/BESIII}

BEPCII is a two-ring $e^{+} e^{-}$collider that operates in the tau-charm energy region $\left(E_{c m}=2.0-\right.$ 4.6 GeV), allowing precision studies of charmonium $\left(J / \psi, \psi^{\prime}, \psi(3770), \eta_{c}, \chi_{c J}\right.$, and $\left.h_{c}\right)$, charm ( $D$ and $D_{s}$ mesons), and improved determinations of the tau mass and the hadronic cross section $(R)$ in this energy region. It has a design luminosity of $1 \times 10^{33} \mathrm{~cm}^{-2} \mathrm{~s}^{-1}$ at a beam energy of 1.89 


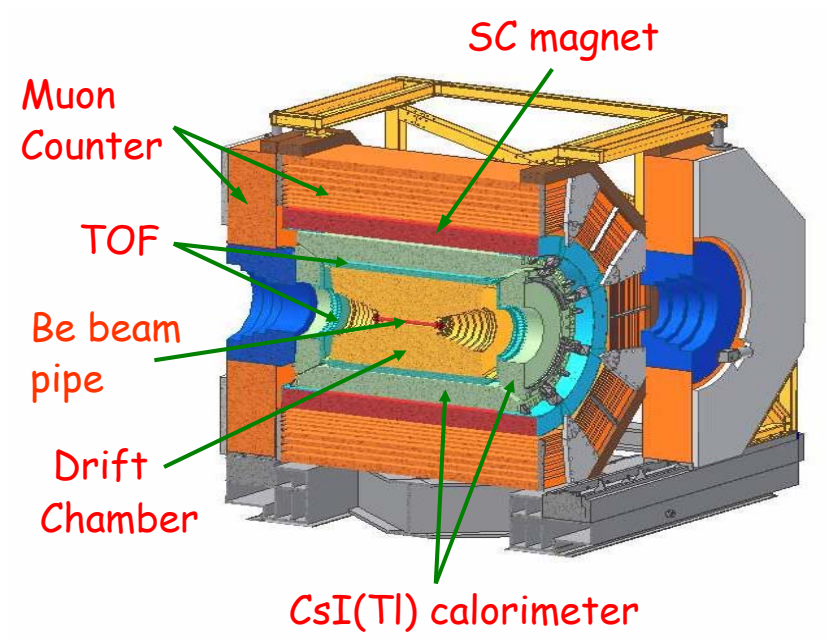

Figure 1: Schematic view of the BESIII detector.

$\mathrm{GeV}$, an improvement of a factor of 100 with respect to the BEPC. This is accomplished mainly by using multi-bunches and super-conducting micro-beta quadrupole magnets.

There are two storage rings with lengths of 237.5 meters. Electrons and positrons collide at the interaction point (IP) with a horizontal crossing angle of $22 \mathrm{mrad}$ and bunch spacing of $8 \mathrm{~ns}$. Each ring has 93 bunches with a design beam current of $910 \mathrm{~mA}$.

The BEPCII collider has operated since 2009. So far, a peak luminosity of $6.5 \times 10^{32} \mathrm{~cm}^{-2} \mathrm{~s}^{-1}$, and beam currents of over $700 \mathrm{~mA}$ for both beams have been achieved.

The BESIII detector consists of a beryllium beam pipe, a helium-based small-celled drift chamber, Time-Of-Flight counters (TOF) for particle identification, a CsI(Tl) crystal calorimeter, a super-conducting solenoidal magnet with a field of 1 Tesla, and a muon identifier using the magnet yoke interleaved with Resistive Plate Counters (RPC). Figure 1 shows the schematic view of the BESIII detector, including both the barrel and end cap portions. Details on BESIII and BEPCII are described in Ref. [5].

\section{Physics results}

Following are physics results based on our $\psi^{\prime}$ and $J / \psi$ data sets. The $\psi^{\prime}$ decays into lower mass charmonium states, including the $h_{c}$, the three $\chi_{c J}$ states, and the $\eta_{c}$, as well as the $J / \psi$, allowing the study of the properties and decays of these states as well as those of the $\psi$ state. For more detail, please see the references.

\section{1 $h_{c}$ studies}

In 2005, CLEO [6] reported a measurement of the mass of the singlet, angular momentum $L=1 h_{c}\left({ }^{1} P_{1}\right)$ in $e^{+} e^{-} \rightarrow \psi^{\prime} \rightarrow \pi^{0} h_{c}, h_{c} \rightarrow \gamma \eta_{c}$, where they used both inclusive and exclusive $\eta_{c}$ decay events. In 2008, they repeated their analysis with $25 \mathrm{M} \psi$ events [7]. Combining results, they obtain $m\left(h_{c}\right)_{A V G}=3525.2 \pm 0.18 \pm 0.12 \mathrm{MeV} / \mathrm{c}^{2}$ [7]. A precise determination of 


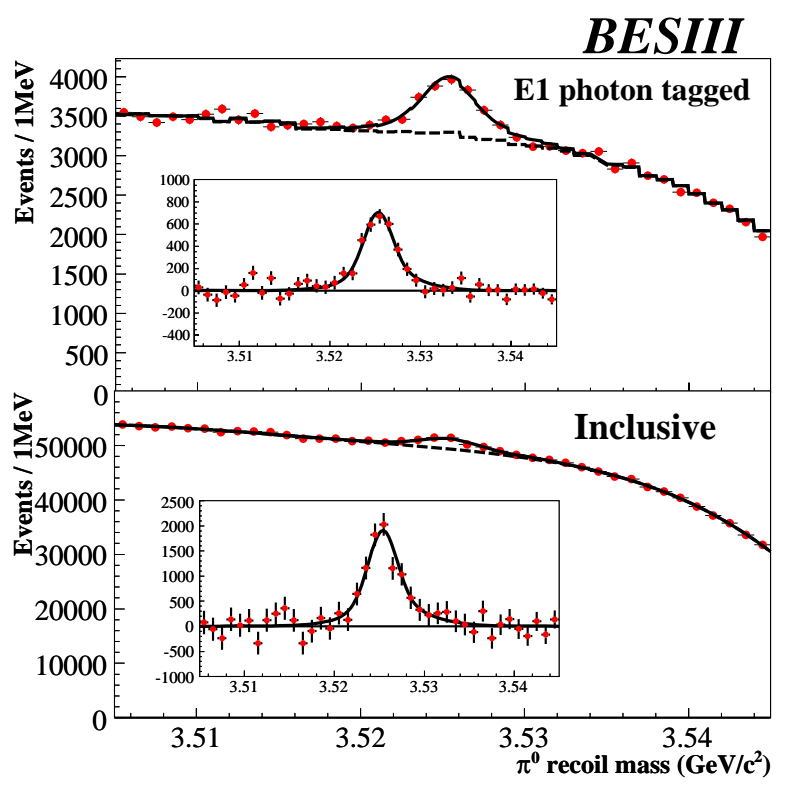

Figure 2: Distributions of mass recoiling against the $\pi^{0}$ in $\psi^{\prime} \rightarrow \pi^{0} h_{c}$ for the sample tagged by the $E 1$ photon from $h_{c} \rightarrow \gamma \eta_{c}$ (top plot), and for inclusive $\psi^{\prime} \rightarrow \pi^{0} h_{c}$ (bottom). Points with error bars are data, and the curves are the best fit results.

the mass is important to learn about the hyperfine (spin-spin) interaction of $P$ wave states. Using the spin weighted centroid of the ${ }^{3} P_{J}$ states, $\left\langle m\left({ }^{3} P_{J}\right)>\right.$, to represent $m\left({ }^{3} P_{J}\right)$, they obtain $\Delta m_{h f}(1 P)=\left\langle m\left({ }^{3} P_{J}\right)>-m\left({ }^{1} P_{1}\right)=+0.08 \pm 0.18 \pm 0.12 \mathrm{MeV} / \mathrm{c}^{2}\right.$. This is consistent with the lowest order expectation of zero.

BESIII has measured $m\left(h_{c}\right)$ from the distribution of mass recoiling against $\pi^{0} \mathrm{~s}$, both in inclusive $\psi^{\prime} \rightarrow \pi^{0} h_{c}$ samples and with samples tagged by the $E 1$ photon from $h_{c} \rightarrow \gamma \eta_{c}$, as shown in Fig. 2. This allows for the first time determinations of $B\left(\psi^{\prime} \rightarrow \pi^{0} h_{c}\right)$ and $B\left(h_{c} \rightarrow \gamma \eta_{c}\right)$ separately. BESIII also measures for the first time the width $\Gamma\left(h_{c}\right)$. Results are shown in Table 1 and compared with CLEOc and theory. More detail may be found in Ref. [8].

Table 1: BESIII $h_{c}$ results compared with CLEOc and theory.

\begin{tabular}{lccc}
\hline & BESIII & CLEOc [7] & Theory \\
\hline$B\left(\psi^{\prime} \rightarrow \pi^{0} h_{c}\right)$ & $4.58 \pm 0.40 \pm 0.50$ & $4.16 \pm 0.30 \pm 0.37$ & \\
$\times B\left(h_{c} \rightarrow \gamma \eta_{c}\right)\left[10^{-4}\right]$ & & & \\
\hline$B\left(\psi^{\prime} \rightarrow \pi^{0} h_{c}\right)\left[10^{-4}\right]$ & $8.4 \pm 1.3 \pm 1.0$ & & $(4-13)[9]$ \\
\hline$B\left(h_{c} \rightarrow \gamma \eta_{c}\right)[\%]$ & $54.3 \pm 6.7 \pm 5.2$ & & (NRQCD) [9] \\
& & & 88 (PQCD) [9] \\
& & 38 [10] \\
\hline$m\left(h_{c}\right)\left[\mathrm{MeV} / \mathrm{c}^{2}\right]$ & $3525.40 \pm 0.13 \pm 0.18$ & $3525.20 \pm 0.18 \pm 0.12$ & \\
\hline$\Gamma\left(h_{c}\right)\left[\mathrm{MeV} / \mathrm{c}^{2}\right]$ & $0.73 \pm 0.45 \pm 0.28$ & & 1.1 (NRQCD) [9] \\
& $<1.44 @ 90 \%$ C.L. & & 0.51 (PQCD) [9] \\
\hline$\Delta M_{h f}(1 P)\left[\mathrm{MeV} / \mathrm{c}^{2}\right]$ & $-0.10 \pm 0.13 \pm 0.18$ & $0.08 \pm 0.18 \pm 0.12$ & \\
\hline
\end{tabular}



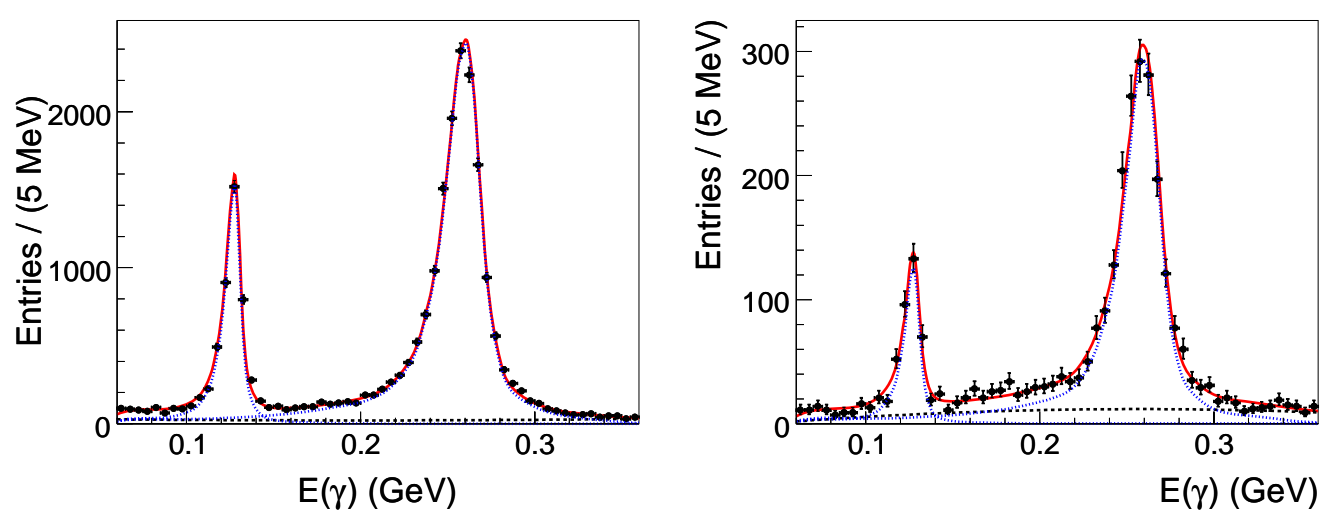

Figure 3: Energy distributions of the radiative photon in $\psi^{\prime} \rightarrow \gamma \chi_{c J}, \chi_{c J} \rightarrow \pi^{0} \pi^{0}$ (left plot) and $\chi_{c J} \rightarrow \eta \eta$ (right plot). Dots with error bars are data. The solid curves are the fit results, and the dotted curves are the $\chi_{c J}$ signals. The dashed curves are the background polynomials.

\section{$3.2 \chi_{c J}$ decays}

The $\chi_{c J}$ states have spin $S=1$ and angular momentum $L=1$, which combine to give total angular momentum $J=0,1$, or 2 . Because of the large $\psi^{\prime}$ sample and large branching fractions $(\sim 10 \%)$ for $\psi^{\prime} \rightarrow \gamma \chi_{c J}$, BESIII is in a good position to study both inclusive and exclusive $\chi_{c J}$ decays. Since $\chi_{c J}$ decays via the production of two gluons, these decays are a good place to search for gluonium [11]. The color octet mechanism has been found to be important in these decays [12], and measurements allow tests of theoretical models.

\subsection{1 $\chi_{c J} \rightarrow \pi^{0} \pi^{0}$ and $\eta \eta$}

BESIII has studied $\psi^{\prime} \rightarrow \gamma \chi_{c J}, \chi_{c J} \rightarrow \pi^{0} \pi^{0}$ and $\eta \eta$, where $\pi^{0}$ and $\eta$ decay to $\gamma \gamma$ [13]. The energy distributions of the candidate radiative photons are shown in Fig. 3. Branching fraction results are given in Table 2 and compared to CLEOc [14], the particle data tables (PDG10) [15], and theory [12]. Improved measurements will allow refinement of theory.

Table 2: Branching fraction results for $\chi_{c J} \rightarrow \pi^{0} \pi^{0}$ and $\eta \eta$. The last errors for BESIII and CLEOc are the branching fraction uncertainties for $\psi^{\prime} \rightarrow \gamma \chi_{c J}$. Note that CLEOc used their own $\psi^{\prime} \rightarrow \gamma \chi_{c J}$ branching fractions for their results.

\begin{tabular}{lccc}
\hline Decay & & $\chi_{c 0}\left(10^{-3}\right)$ & $\chi_{c 2}\left(10^{-3}\right)$ \\
\hline$\pi^{0} \pi^{0}$ & BESIII [13] & $3.23 \pm 0.03 \pm 0.23 \pm 0.14$ & $0.88 \pm 0.02 \pm 0.06 \pm 0.04$ \\
& CLEOc [14] & $2.94 \pm 0.07 \pm 0.32 \pm 0.15$ & $0.68 \pm 0.03 \pm 0.07 \pm 0.04$ \\
& PDG10 [15] & $2.80 \pm 0.13$ & $0.80 \pm 0.05$ \\
& Theory [12] & 2.3 & 0.95 \\
\hline$\eta \eta$ & BESIII [13] & $3.44 \pm 0.10 \pm 0.24 \pm 0.20$ & $0.65 \pm 0.04 \pm 0.05 \pm 0.03$ \\
& CLEOc [14] & $3.18 \pm 0.13 \pm 0.31 \pm 0.16$ & $0.51 \pm 0.05 \pm 0.05 \pm 0.03$ \\
& PDG10 [15] & $2.68 \pm 0.28$ & $0.54 \pm 0.08$ \\
& Theory [12] & 3.2 & 1.3 \\
\hline
\end{tabular}




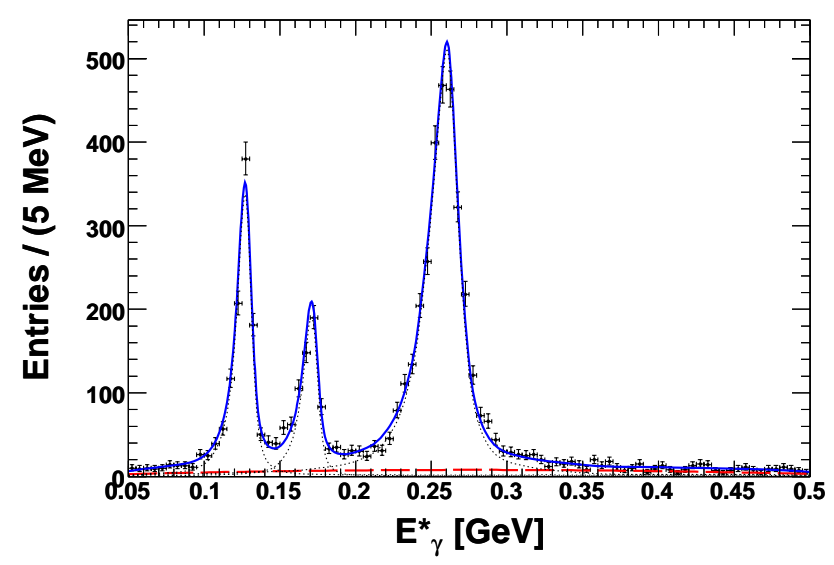

Figure 4: Energy distribution of the radiative photon in $\psi^{\prime} \rightarrow \gamma \chi_{c J}, \chi_{c J} \rightarrow 4 \pi^{0}$, where clear peaks are observed, corresponding from left to right to the $\chi_{c 2}, \chi_{c 1}$, and the $\chi_{c 0}$.

\subsection{2 $\chi_{c J} \rightarrow 4 \pi^{0}$}

The largest $\chi_{c J}$ branching fractions are $\chi_{c J} \rightarrow 4 \pi$ decays. The branching fractions for $\chi_{c J} \rightarrow$ $\pi^{+} \pi^{-} \pi^{+} \pi^{-}$and $\pi^{+} \pi^{-} \pi^{0} \pi^{0}$ have been measured, but the $\chi_{c J} \rightarrow 4 \pi^{0}$ branching fractions have not been. BESIII has now measured the branching fractions for $\chi_{c J} \rightarrow 4 \pi^{0}$. Nine photons are required, from which $4 \pi^{0} \mathrm{~s}$ are selected. To improve the mass resolution and remove background, a four constraint kinematic fit is performed under the hypothesis of $\psi \rightarrow 9 \gamma$, and $\chi^{2}<15$ is required. The energy distribution of the candidate radiative photon is shown in Fig. 4, where three clear peaks are observed, corresponding to the $\chi_{c 2}, \chi_{c 1}$, and the $\chi_{c 0}$. Branching fractions excluding intermediate $K_{S} \rightarrow \pi^{0} \pi^{0}$ are listed in Table 3. These are first measurements. For more detail, see Ref. [16].

Table 3: Branching fraction results for $\chi_{c J} \rightarrow 4 \pi^{0}$.

\begin{tabular}{lc}
\hline Decay & Branching Fraction $\left(10^{-3}\right)$ \\
\hline$\chi_{c 0} \rightarrow 4 \pi^{0}$ & $3.34 \pm 0.06 \pm 0.44$ \\
$\chi_{c 1} \rightarrow 4 \pi^{0}$ & $0.57 \pm 0.03 \pm 0.08$ \\
$\chi_{c 2} \rightarrow 4 \pi^{0}$ & $1.21 \pm 0.05 \pm 0.16$ \\
\hline
\end{tabular}

\subsection{3 $\chi_{c J} \rightarrow \gamma V$}

BESIII has studied $\psi^{\prime} \rightarrow \gamma \chi_{c J}, \chi_{c J} \rightarrow \gamma V$, where $V$ is $\phi \rightarrow K^{+} K^{-}, \rho \rightarrow \pi^{+} \pi^{-}$, or $\omega \rightarrow$ $\pi^{+} \pi^{-} \pi^{0}$. Invariant mass distributions of the high energy gamma and $V$ are shown in Fig. 5, where clear signals for $\chi_{c 1} \rightarrow \gamma V$ are observed. Results are given in Table 4 and compared to CLEOc [17] and perturbative QCD predictions [18], which for $\chi_{c 1} \rightarrow \gamma V$ are an order of magnitude too low. By including hadronic loop effects into the perturbative QCD predictions, Ref. [19] can accommodate the larger experimental values. BESIII observes $\chi_{c 1} \rightarrow \gamma \phi$ for the first time.

BESIII has also fit the helicity angle distributions in the decays $\chi_{c 1} \rightarrow \gamma V$, shown in Fig. 6. In $\chi_{c 1} \rightarrow \gamma V$ decays, the final state is a superposition of longitudinal $(\lambda=0)$ and transverse $(\lambda= \pm 1)$ 


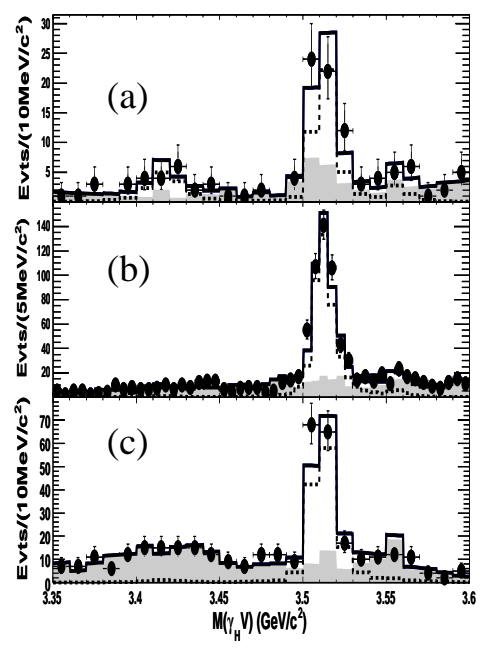

Figure 5: Invariant mass distributions of the high energy gamma and $V$ from $\psi^{\prime} \rightarrow \gamma \chi_{c J}, \chi_{c J} \rightarrow \gamma V$, where $V$ is (a) $\phi$, (b) $\rho$, or (c) $\omega$. Dots with error bars are data, the black histograms are the best fits, the dashed histograms are the signal shapes, and the shaded histograms are the sum of the sideband background and the background polynomials. Clear signals for $\chi_{c 1} \rightarrow \gamma V$ are observed.

Table 4: Branching fraction results for $\chi_{c J} \rightarrow \gamma V$, where $V$ is a $\phi, \rho$, or $\omega$. Upper limits are at the $90 \%$ confidence level.

\begin{tabular}{lccc}
\hline Decay & BESIII $\left(10^{-6}\right)$ & CLEOc [17] $\left(10^{-6}\right)$ & pQCD [18] $\left(10^{-6}\right)$ \\
\hline$\chi_{c 0} \rightarrow \gamma \phi$ & $<16.2$ & $<6.4$ & 0.46 \\
$\chi_{c 1} \rightarrow \gamma \phi$ & $25.8 \pm 5.2 \pm 2.3$ & $<26$ & 3.6 \\
$\chi_{c 2} \rightarrow \gamma \phi$ & $<8.1$ & $<13$ & 1.1 \\
\hline$\chi_{c 0} \rightarrow \gamma \rho^{0}$ & $<10.5$ & $<9.6$ & 1.2 \\
$\chi_{c 1} \rightarrow \gamma \rho^{0}$ & $228 \pm 13 \pm 22$ & $243 \pm 19 \pm 22$ & 14 \\
$\chi_{c 2} \rightarrow \gamma \rho^{0}$ & $<20.8$ & $<50$ & 4.4 \\
\hline$\chi_{c 0} \rightarrow \gamma \omega$ & $<12.9$ & $<8.8$ & 0.13 \\
$\chi_{c 1} \rightarrow \gamma \omega$ & $69.7 \pm 7.2 \pm 6.6$ & $83 \pm 15 \pm 12$ & 1.6 \\
$\chi_{c 2} \rightarrow \gamma \omega$ & $<6.1$ & $<7.0$ & 0.5
\end{tabular}

polarizations. The angular distribution is

$$
\frac{d \Gamma}{\Gamma d \cos \theta} \propto\left(1-f_{T}\right) \cos ^{2} \Theta+\frac{1}{2} f_{T} \sin ^{2} \Theta
$$

where $f_{T}=\left|A_{T}\right|^{2} /\left(\left|A_{T}\right|^{2}+\left|A_{L}\right|^{2}\right)$ is the transverse polarization fraction in the decay and $A_{L}$ and $A_{T}$ are the longitudinal and transverse polarization amplitudes, respectively, and the helicity angle $\Theta$ is the angle between the vector meson flight direction in the $\chi_{c 1}$ rest frame and either the $\pi^{+} / K^{+}$ direction in the $\rho^{0} / \phi$ rest frame or the normal to the $\omega$ decay plane in the $\omega$ rest frame. Results for $f_{t}$ are given in Table 5, along with results from CLEOc [17], where we have used CLEO-c's $A_{ \pm} / A_{0}$ to determine $f_{t}$. The results are consistent within $2 \sigma$. The helicity distributions indicate that the vector mesons are preferentially longitudinally polarized. More detail may be found in Ref. [20]. 


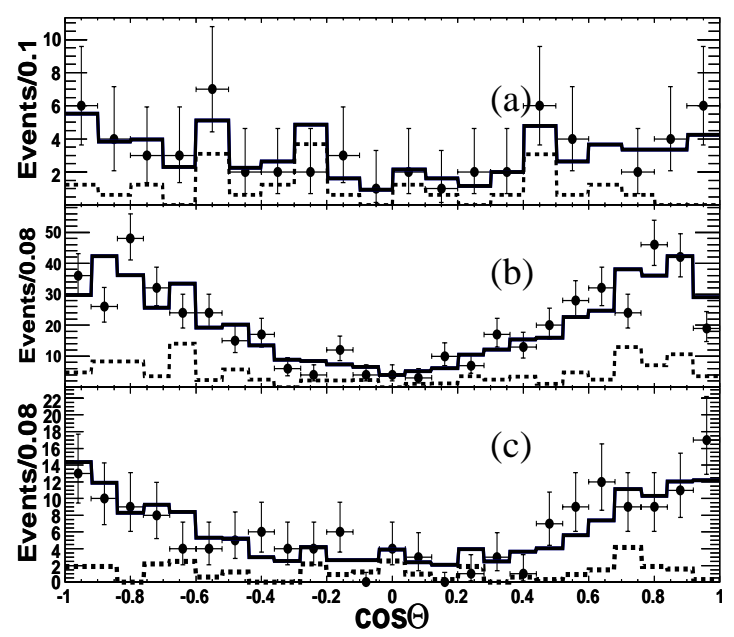

Figure 6: $\cos \Theta$ distributions and the fits for (a) $\chi_{c 1} \rightarrow \gamma \phi$, (b) $\chi_{c 1} \rightarrow \gamma \rho^{0}$, and (c) $\chi_{c 1} \rightarrow \gamma \omega$. Dots with error bars are data; the solid histograms are the fits; and dashed histograms are the sums of the sideband background and the polynomial background.

Table 5: Results of fits to helicity angle distributions in $\chi_{c 1} \rightarrow \gamma V . \quad f_{T}=\left|A_{T}\right|^{2} /\left(\left|A_{T}\right|^{2}+\left|A_{L}\right|^{2}\right)$ is the transverse polarization fraction in the decay, and $A_{L}$ and $A_{T}$ are the longitudinal and transverse polarization amplitudes, respectively.

\begin{tabular}{lcc}
\hline Decay & $f_{t}$-BESIII & $f_{t}$-CLEOc [17] \\
\hline$\chi_{c 1} \rightarrow \gamma \rho$ & $0.158 \pm 0.034_{-0.014}^{+0.015}$ & $0.072_{-0.031-0.019}^{+0.041+0.002}$ \\
$\chi_{c 1} \rightarrow \gamma \omega$ & $0.247_{-0.087-0.026}^{+0.090+0.044}$ & $0.32_{-0.11-0.11}^{+0.17+0.05}$ \\
$\chi_{c 1} \rightarrow \gamma \phi$ & $0.29_{-0.13+-0.09}^{+0.10}$ & \\
\hline
\end{tabular}

\section{$3.3 X(1860)$ and $X(1835)$}

BESII observed a $p \bar{p}$ threshold enhancement in $J / \psi \rightarrow \gamma p \bar{p}$ [21]. Fitted to an $S$-wave resonance, it gives a mass below $p \bar{p}$ threshold of $M(p \bar{p})=1859_{-10-25}^{+3+5} \mathrm{MeV} / c^{2}$. The observation of the $X(1860)$ has generated much interest, partly because the possibility of a $p \bar{p}$ bound state, baryonium, was first suggested by E. Fermi and C. N. Yang in 1949 [22].

BESIII has confirmed this observation in $\psi^{\prime} \rightarrow \pi^{+} \pi^{-} J / \psi, J / \psi \rightarrow \gamma p \bar{p}$, as shown in Fig. 7 a, where it obtains a fitted mass of $M(p \bar{p})=1861_{-13-26}^{+6+7} \mathrm{MeV} / c^{2}$ and a width $\Gamma<38 \mathrm{MeV} / c^{2}$ at the $90 \%$ confidence level (C.L.) [23]. BESIII also confirms it directly in a preliminary study of $J / \psi \rightarrow \gamma p \bar{p}$, shown in Fig. $7 \mathrm{~b}$, with a fitted mass of $M(p \bar{p})=1861.6 \pm 0.8 \mathrm{MeV} / c^{2}$, where the error is only the statistical error, and a narrow width that is $\Gamma<8 \mathrm{MeV} / c^{2}$ at the $90 \%$ C.L.. CLEOc also observes a $p \bar{p}$ threshold enhancement in $\psi^{\dagger} \rightarrow \pi^{+} \pi^{-} J / \psi, J / \psi \rightarrow \gamma p \bar{p}$ and obtains a consistent fitted mass [24].

BESII also observed a resonance at $M\left(\eta^{\prime} \pi^{+} \pi^{-}\right)=1833.7 \pm 6.1 \pm 2.7 \mathrm{MeV} / c^{2}$ in $J / \psi \rightarrow$ $\gamma \eta^{\prime} \pi^{+} \pi^{-}$where $\eta^{\prime} \rightarrow \pi^{+} \pi^{-} \eta$ and $\gamma \rho$ [25]. After the observation of the $X(1860)$, it was suggested that BES look in this decay mode, since $p \bar{p}$ is rich in gluon content and $\eta$ has a strong coupling to gluons [26]. BESIII also confirms this observation [27], shown in Fig. 8. However, there are two additional high mass peaks, as well as an $f_{1}(1510)$ at lower mass. Fitting all mass peaks, 

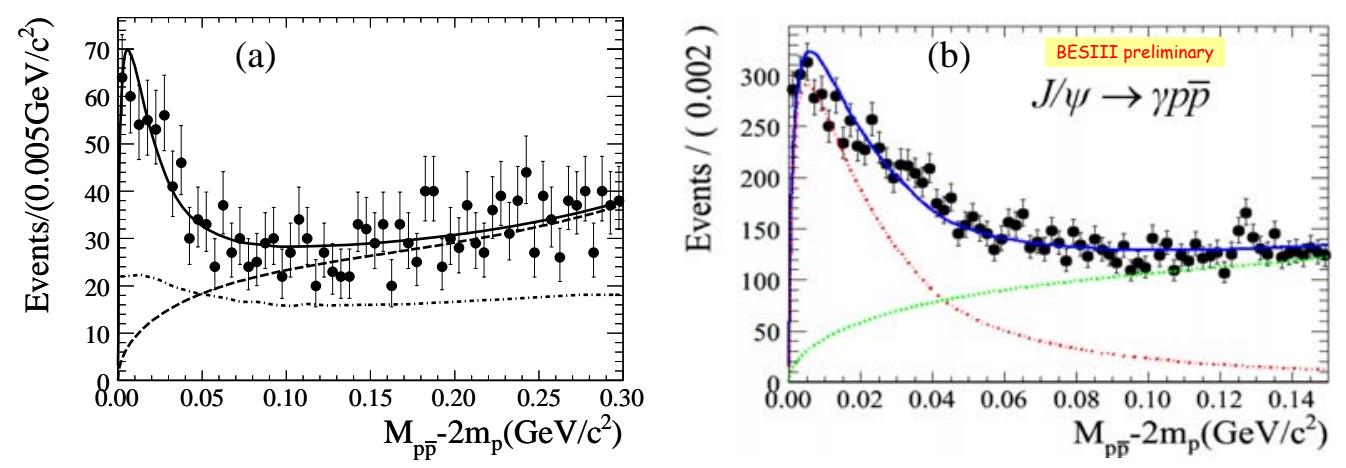

Figure 7: a.) The $p \bar{p}$ threshold enhancement in $\psi^{\prime} \rightarrow \pi^{+} \pi^{-} J / \psi, J / \psi \rightarrow \gamma p \bar{p}$ [23]. The solid curve is the fit result, the dashed curve is the fitted background function, and the dash-dotted curve shows how the acceptance varies with $p \bar{p}$ mass. b.) The $p \bar{p}$ threshold enhancement directly in $J / \psi \rightarrow \gamma p \bar{p}$. The solid curve is the fit result, the dashed curve is the fitted background function, and the dash-dotted curve shows the signal shape. The result is preliminary.

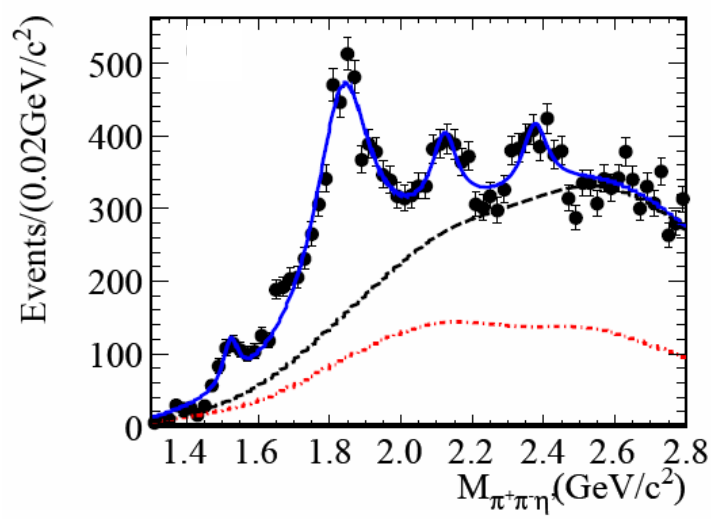

Figure 8: The $\eta^{\prime} \pi^{+} \pi^{-}$invariant mass distribution in $J / \psi \rightarrow \gamma \eta^{\prime} \pi^{+} \pi^{-}$where $\eta^{\prime} \rightarrow \pi^{+} \pi^{-} \eta$ and $\gamma \rho$. The dash-dot curve corresponds to contributions from non- $\eta^{\prime}$ events and the $\pi^{0} \pi^{+} \pi^{-} \eta^{\prime}$ backgrounds for the two decay modes, and the dashed line to contributions of the total background and the non-resonant $\pi^{+} \pi^{-} \eta^{\prime}$ process.

we obtain masses of $M(X(1835))=1836.5 \pm 3.0_{-2.1}^{+5.6} \mathrm{MeV} / c^{2}, M(X(2120))=2122.4 \pm 6.7_{-2.7}^{+4.7}$ $\mathrm{MeV} / \mathrm{c}^{2}$, and $M(X(2370))=2376.3 \pm 8.7_{-4.3}^{+3.2} \mathrm{MeV} / \mathrm{c}^{2}$ and widths of $\Gamma(X(1835))=190 \pm 9_{-36}^{+38}$ $\mathrm{MeV} / c^{2}, \Gamma(X(2120))=83 \pm 16_{-11}^{+31} \mathrm{MeV} / c^{2}$, and $\Gamma(X(2370))=83 \pm 17_{-6}^{+44} \mathrm{MeV} / c^{2}$.

For radiative decays to a pseudoscalar meson, the polar angle of the photon in the $J / \psi$ center of mass system, $\theta_{\gamma}$, should be distributed according to $1+\cos ^{2} \theta_{\gamma}$. The background-subtracted, acceptance-corrected $\left|\cos _{\gamma}\right|$ distribution of the $X(1835)$ is consistent with this distribution, and $X(1835)$ is consistent with being a pseudoscalar meson. However, more data is required to complete a full partial wave analysis of these states. More detail may be found in Ref. [27]. 

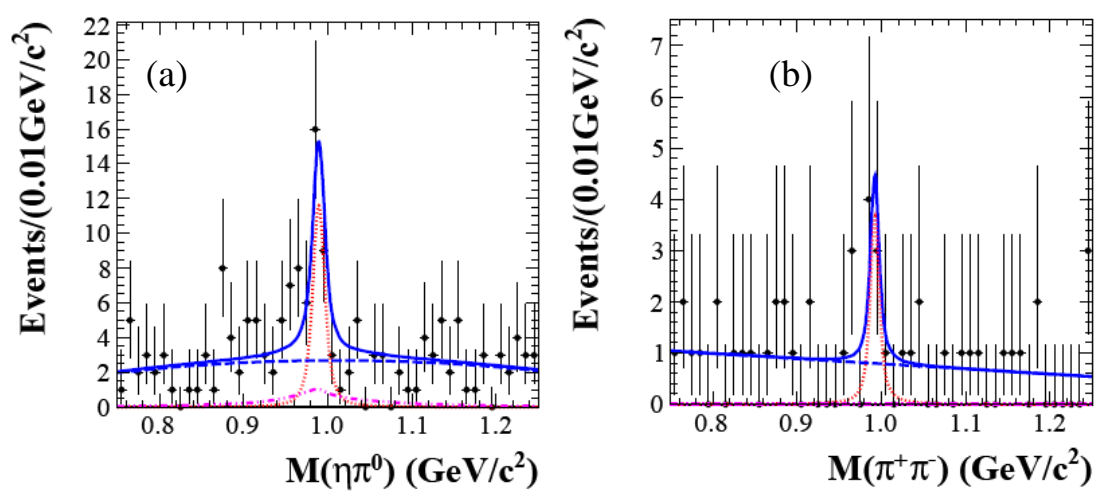

Figure 9: a.) Invariant mass of $\eta \pi^{0}$ in $J / \psi \rightarrow \phi f_{0} \Rightarrow \phi a_{0} \rightarrow \phi \eta \pi^{0}$. The solid curve is the fit, the dotted curve is the very narrow $\left(<8 \mathrm{MeV} / \mathrm{c}^{2}\right)$ mixing signal, and the dash-dot curve is the wide $a_{0}^{0}(980)$ contribution from $\gamma^{*}$ or $K^{*} K$ loops. b.) Invariant mass spectrum of $\pi^{+} \pi^{-}$in $\chi_{c 1} \rightarrow \pi^{0} a_{0} \Rightarrow \pi^{0} f_{0} \rightarrow \pi^{+} \pi^{-} \pi^{0}$. The solid curve is the fit result, the dotted curve is the mixing signal, and the dash-dotted curve indicates the $f_{0}(980)$ from other sources. The dashed curve in both figures denotes the polynomial background.

\section{$3.4 f_{0}(980)-a_{0}(980)$ mixing}

The $f_{0}(980)$ and $a_{0}(980)$ are controversial particles. They have been described as $q \bar{q}$ or $q \bar{q} q \bar{q}$ states, $K \bar{K}$ molecules, or $q \bar{q} G$ hybrid states. Mixing was first suggested by Achasov [28], and mixing measurements may help to clarify the nature of these particles. There have been suggestions for BESIII to search for mixing signals in $J / \psi \rightarrow \phi f_{0} \Rightarrow \phi a_{0} \rightarrow \phi \eta \pi^{0}$ [29], where the $f_{0}$ mixes to $a_{0}$, and in the process $\chi_{c 1} \rightarrow \pi^{0} a_{0} \Rightarrow \pi^{0} f_{0} \rightarrow \pi^{+} \pi^{-} \pi^{0}$ [30], where $a_{0}$ mixes to $f_{0}$. The signal for the former case is shown in Fig. 9 a). The expected mixing signal is very narrow $\left(8 \mathrm{MeV} / c^{2}\right)$, between the $K^{+} K^{-}$and $K_{S}^{0} K_{S}^{0}$ thresholds $\left(987-995 \mathrm{MeV} / c^{2}\right)$. Backgrounds are sideband and a wide $a_{0}$ from $J / \psi \rightarrow \gamma^{*} / K^{*} K \rightarrow \phi a_{0}$. Fitting to signal plus backgrounds determines $24.7 \pm 8.6$ mixing events or $<36.7$ at the $90 \%$ C.L. This gives a mixing intensity $\xi_{f a}=\frac{B\left(J / \psi \rightarrow \phi f_{0} \Rightarrow \phi a_{0} \rightarrow \phi \eta \pi^{0}\right)}{B\left(J / \psi \rightarrow \phi f_{0} \rightarrow \phi \pi^{+} \pi^{-}\right)}=$ $(0.60 \pm 0.20 \pm 0.12 \pm 0.26) \%$, where the first error is statistical, the second systematic, and the third is the uncertainty associated with the shape of the mixing signal, or $<1.1 \%$ at the $90 \%$ C.L.

The signal for latter case is shown in Fig. 9 b). The expected mixing signal here is also very narrow. Backgrounds are sideband and a wide $f_{0}$ from other background processes. Fitting to the narrow signal plus backgrounds determines $6.4 \pm 3.2$ mixing events or $<13$ at the $90 \%$ C.L.. We determine $\xi_{f a}=\frac{B\left(\chi_{c 1} \rightarrow a_{0} \pi^{0} \Rightarrow f_{0} \pi^{0} \rightarrow \pi^{+} \pi^{-} \pi^{0}\right)}{B\left(\chi_{c 1} \rightarrow a_{0}(980) \pi^{0} \rightarrow \eta \pi^{0} \pi^{0}\right)}=(0.31 \pm 0.16 \pm 0.14 \pm 0.03) \%$, where the first error is statistical, the second systematic, and the third is the uncertainty associated with the shape of the mixing signal, or $<1.0 \%$ at the $90 \%$ C.L. Figure 10 shows our results and upper limits compared to models $\left(q \bar{q}, q^{2} \bar{q}^{2}, K \bar{K}\right.$, and $q \bar{q} g$ ) and calculated values (SND, KLOE, BNL, and CB) based on $f_{0}$ and $a_{0}$ resonance parameters. References for models and calculations may be found in Refs. [29] and [30]. Ours are the first direct measurements. More detail may be found in Ref. [31].

\section{$3.5 \psi^{\prime} \rightarrow \gamma P$}

Another channel studied is $\psi^{\prime} \rightarrow \gamma P$, where the pseudoscalar $P$ can be a $\pi^{0}, \eta$, or $\eta^{\prime}$. This process is important for testing various phenomenological mechanisms, including Vector Meson 


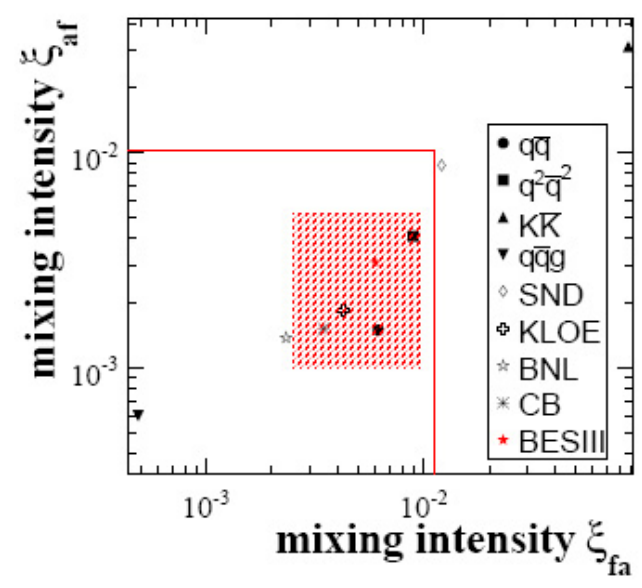

Figure 10: Mixing intensity $\xi_{f a}$ versus $\xi_{a f}$ for various models and calculations and our results (shaded region) and upper limits (lines).

Dominance [32, 33, 34], $\eta_{c}-\eta^{\prime}$ mixing [35, 36], two gluon couplings to $q \bar{q}$ states [33], and final state radiation by light quarks [32]. The value of $R_{J / \psi}=\frac{B(J / \psi \rightarrow \gamma \eta)}{B\left(J / \psi \rightarrow \gamma \eta^{\prime}\right)}$ is predicted in first order perturbation theory [32], and $R_{\psi^{\prime}}=\frac{B\left(\psi^{\prime} \rightarrow \gamma \eta\right)}{B\left(\psi^{\prime} \rightarrow \gamma \eta^{\prime}\right)}=R_{J / \psi}$ is expected [37]. The branching fraction $B\left(\psi^{\prime} \rightarrow \gamma \pi^{0}\right)$ is expected to be small [38].

Recently CLEOc reported results on $J / \psi, \psi^{\prime}, \psi^{\prime \prime} \rightarrow \gamma P$ [37]. They found no evidence for $\psi^{\prime} \rightarrow$ $\gamma \pi^{0}$ or $\gamma \eta$ and set upper limits at the $90 \%$ C.L. of $B\left(\psi^{\prime} \rightarrow \gamma \pi^{0}\right)<5 \times 10^{-6}$ and $R_{\psi^{\prime}}<1.8 \%$. They obtain $R_{J / \psi}=(21.1 \pm 0.9) \%$, and the observation that $R_{\psi^{\prime}}<<R_{J / \psi}$ poses a significant challenge to theory.

BESIII has also studied $\psi^{\prime} \rightarrow \gamma \pi^{0}$ with $\pi^{0} \rightarrow \gamma \gamma, \psi^{\prime} \rightarrow \gamma \eta$ with $\eta \rightarrow \pi^{+} \pi^{-} \pi^{0}$ and $3 \pi^{0}$, and $\psi^{\prime} \rightarrow \gamma \eta^{\prime}$ with $\eta^{\prime} \rightarrow \pi^{+} \pi^{-} \gamma$ and $\pi^{+} \pi^{-} \eta$ and $\eta \rightarrow \gamma \gamma$. Invariant mass distributions showing the signals in all final states are shown in Fig. 11, and branching fractions are listed in Table 6. BESIII measures branching fractions for $\psi^{\prime} \rightarrow \gamma \pi^{0}$ and $\gamma \eta$ for the first time, provides the first measurement of $R_{\psi^{\prime}}=1.10 \pm 0.38 \pm 0.07$, and finds that $R_{\psi^{\prime}}<<R_{J / \psi}$. More detail is provided in Ref. [39].

Table 6: Branching fraction results for $\psi^{\prime} \rightarrow \gamma P$, where $P=\pi^{0}, \eta$, and $\eta^{\prime}$.

\begin{tabular}{lccc}
\hline Decay & BESIII $\left(10^{-6}\right)$ & combined BESIII $\left(10^{-6}\right)$ & PDG [15] $\left(10^{-6}\right)$ \\
\hline$\psi^{\prime} \rightarrow \gamma \pi^{0}$ & $1.58 \pm 0.40 \pm 0.13$ & $1.58 \pm 0.40 \pm 0.13$ & $<5$ \\
$\psi^{\prime} \rightarrow \gamma \eta\left(\pi^{+} \pi^{-} \pi^{0}\right)$ & $1.78 \pm 0.72 \pm 0.17$ & & \\
$\psi^{\prime} \rightarrow \gamma \eta\left(3 \pi^{0}\right)$ & $1.07 \pm 0.65 \pm 0.08$ & $1.38 \pm 0.48 \pm 0.09$ & $<2$ \\
\hline$\psi^{\prime} \rightarrow \gamma \eta^{\prime}\left(\pi^{+} \pi^{-} \eta\right)$ & $120 \pm 5 \pm 8$ & & \\
$\psi^{\prime} \rightarrow \gamma \eta^{\prime}\left(\pi^{+} \pi^{-} \gamma\right)$ & $129 \pm 3 \pm 8$ & $126 \pm 3 \pm 8$ & $121 \pm 8$
\end{tabular}

\section{Beam energy measurement system (BEMS)}

The most precise determination of the mass of the tau lepton, $m_{\tau}=1776.69_{-0.19}^{+0.17} \pm 0.15$ $\mathrm{MeV} / \mathrm{c}^{2}$, was performed [40] by the KEDR experiment at the Budker Institute of Nuclear Physics. The value is consistent with the 1996 measurement by BES, $m_{\tau}=1776.96_{-0.21-0.17}^{+0.18+0.25} \mathrm{MeV} / c^{2}$ [41]. 


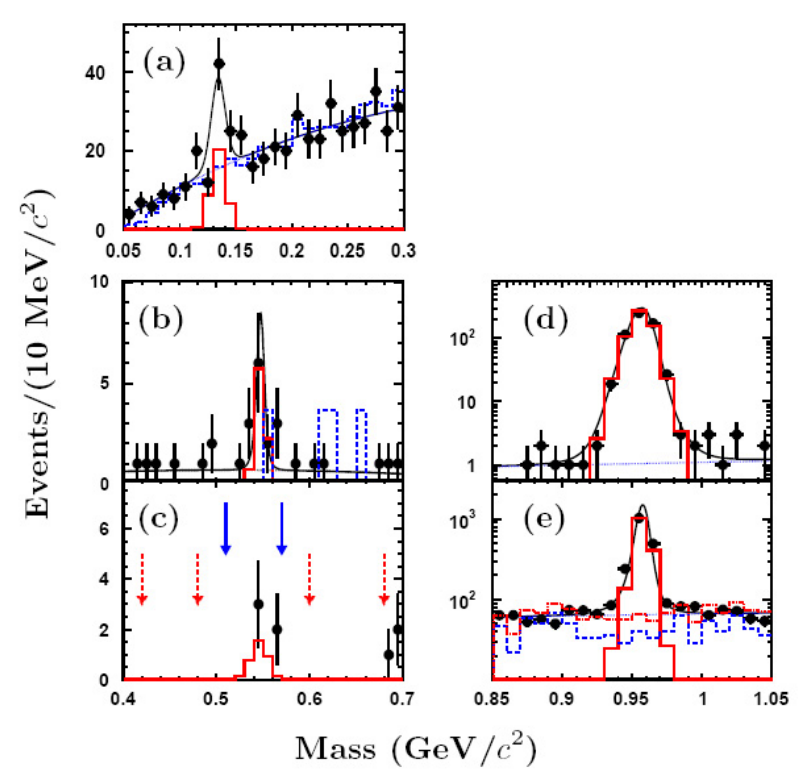

Figure 11: Invariant mass distributions of the pseudoscalar meson candidates in $\psi^{\prime} \rightarrow \gamma P$ : (a) $\gamma \pi^{0}$, (b) $\gamma \eta\left(\pi^{+} \pi^{-} \pi^{0}\right)$, (c) $\gamma \eta\left(3 \pi^{0}\right)$, (d) $\gamma \eta^{\prime}\left(\pi^{+} \pi^{-} \eta\right)$, and (e) $\gamma \eta^{\prime}\left(\gamma \pi^{+} \pi^{-}\right)$. The crosses are data, the solid histograms are MC simulated signal, and the dashed lines are continuum backgrounds. In (c), the arrows indicate the selection region, while the dashed arrows show sideband background regions. In (e), the dotdot-dashed line shows the sum of continuum background and expected background from $\psi^{\prime}$ decays.

The current combined value given by PDG10 is $m_{\tau}=1776.82 \pm 0.16 \mathrm{MeV} / c^{2}$ [15]. Very important in the KEDR measurement was the precise measurement of the beam energy; two methods were used, the resonant spin depolarization technique with a precision of about $30 \mathrm{keV}$ and the back scattered Compton photon technique with a precision of about $60 \mathrm{keV}$ [40]. BESIII will use the later technique.

Improving the precision on the tau mass, which is a fundamental parameter of the Standard Model, is very important. While the masses of the electron and muon are known with a precision of $\frac{\delta M}{M}=10^{-8}$, the mass of the tau is only known to about $10^{-4}$. The test of lepton universality for the muon and tau depends on the mass of the tau to the fifth power. Currently muon-tau universality is tested at the $0.2 \%$ level [42], and this can be improved by a more precise measurement of the mass of the tau lepton.

BESIII will improve the precision of the tau mass, and a beam energy measurement system using back scattered Compton photons will be central to this measurement. The method:

- Photons from a Coherent $\mathrm{CO}_{2}$ laser are directed into the on-coming electron or positron beam, and back scattered Compton photons are detected in a High Purity Germanium detector (HPGe).

- The beam energy $\varepsilon$ is determined by the maximum energy of the back scattered photons $\left(\omega_{\max }\right)$ by

$$
\varepsilon=\frac{\omega_{\max }}{2}\left[1+\sqrt{1+\frac{m_{e}^{2}}{\omega_{0} \omega_{\max }}}\right]
$$




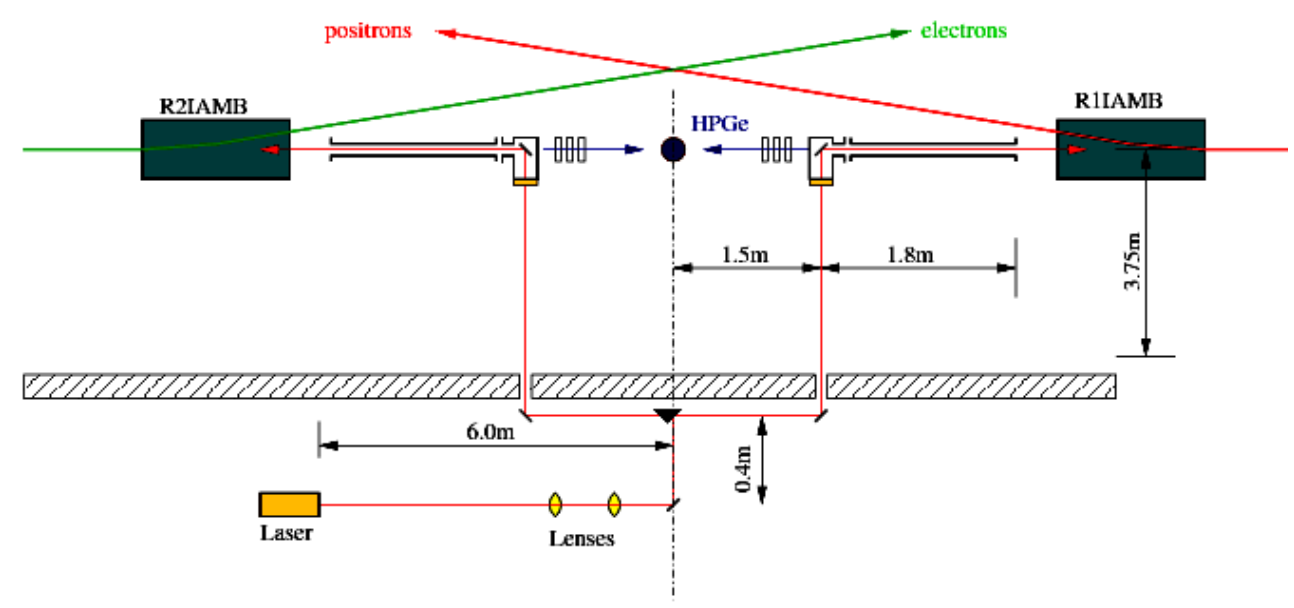

Figure 12: Schematic of the beam energy measurement system at BESIII.

where $\omega_{0}$ is the energy of the incident photon.

- The energies of the back scattered photons are measured in a HPGe detector with precision of $\delta \omega / \omega \sim 1 \times 10^{-5}$.

- The absolute energy scale is calibrated with $\gamma$-active radionuclides.

- The mass of the tau is expected to be determined to better than $100 \mathrm{keV}$ in a data taking run of about two weeks.

The schematic of the method is shown in Fig. 12. The $\mathrm{CO}_{2}$ laser beam is focused and directed to a movable prism by a $45^{\circ}$ mirror. The position of the prism determines which beam is illuminated. The light is then reflected into the oncoming electron or positron beam by a copper mirror inside an extension to the vacuum enclosure. The copper mirror is transparent to the Compton back scattered photons, which are then detected in the HPGe.

Figure 13 a shows the vacuum pipe extension containing a GaAs window to pass the laser light and the copper mirror. The arrows indicate the directions of the incoming laser light and the back scattered Compton photons.

Figure $13 \mathrm{~b}$ shows the energy distribution of the Compton back scattered photons; the fit to determine the maximum back scattered energy is shown. This data is from a test run of the system at the end of 2010 .

\section{Present and future program}

BEPCII is currently running at the $\psi^{\prime \prime}$, which is above the threshold for open charm. The $\psi^{\prime \prime}$ mostly decays into correlated $D \bar{D}$ pairs, allowing the study of quantum correlations. Since the $D$ mesons are always produced in pairs, this is an excellent laboratory for making absolute measurements of $D$ branching fractions, searching for rare $D$ decays, studying $D-\bar{D}$ mixing, searching 


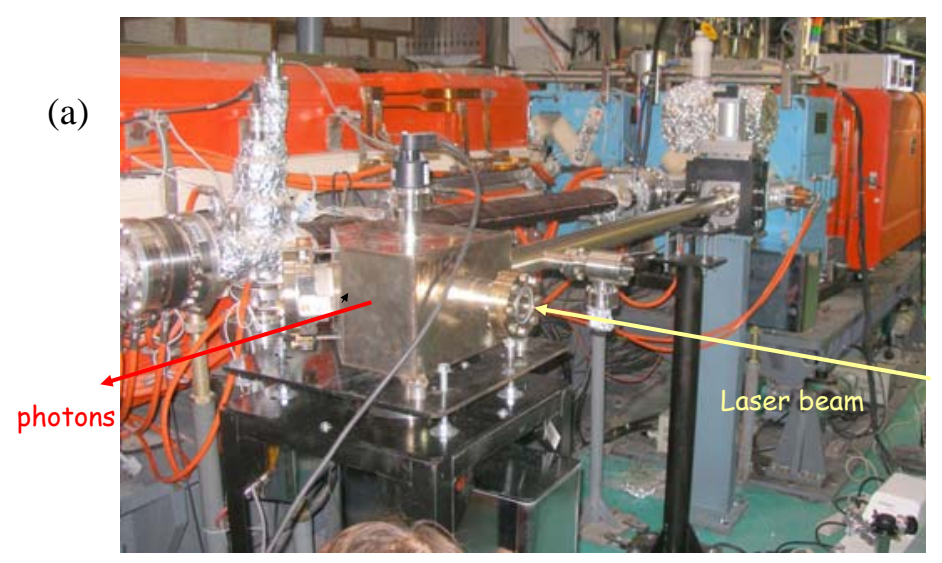

(b)

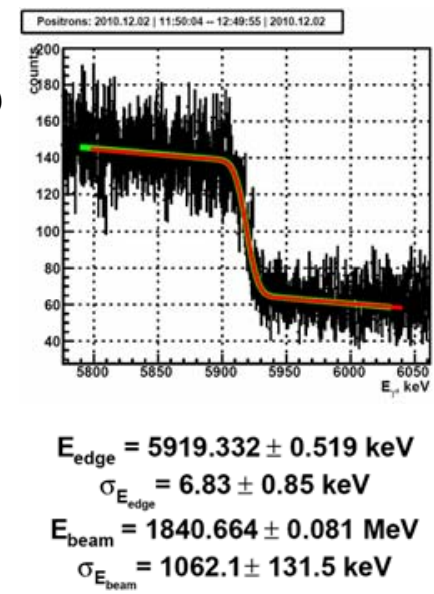

Figure 13: (a) The vacuum pipe extension containing a GaAs window to pass the laser light and the copper mirror. The arrows indicate the directions of the incoming laser light and the back scattered Compton photons. (b) Energy distribution of the Compton back scattered photons; the fit to determine the maximum back scattered energy is shown. This data is from a test run of the system at the end of 2010.

for $C P$ violation, and measuring form factors in $D$ leptonic decays and structure functions in $D$ semileptonic decays. The measurements will provide calibrations for lattice QCD. At present, the goal is to accumulate about $2 \mathrm{fb}^{-1}$ of $\psi^{\prime \prime}$ data giving BESIII a total including an earlier run of approximately $3 \mathrm{fb}^{-1}$.

After reaching this goal, BESIII will move up in energy and accumulate a sample at a center of mass energy of $4010 \mathrm{MeV} / \mathrm{c}^{2}$. Here it will look for transitions to lower mass states.

BEPCII and BESIII will operate for many years. Running will include more $J / \psi$ and $\psi^{\dagger}$ data, as well as a tau mass scan in order to make a more precise determination of the tau mass. Running at $4010 \mathrm{MeV} / c^{2}$ and above will allow studies of $D_{S}$ and $D^{*}$ mesons in a similar fashion to the study of $D$ mesons at the $\psi^{\prime \prime}$.

Also detailed energy scans will allow improvement in the determination of $R=\frac{\sigma\left(e^{+} e^{-} \rightarrow \text { hadrons }\right)}{\sigma\left(e^{+} e^{-} \rightarrow \mu^{+} \mu^{-}\right)}$ as a function of energy. More precise $R$ measurements are extremely important to improve the predicted value of $a_{\mu}$, important for testing the standard model prediction of $g-2$, and to determine the fine structure constant $\alpha$ at the mass of the $Z$ boson, which is important in tests of the standard model and in predicting the mass of the Higgs. Measurements of $R$ in the charm resonance region will allow better understanding of the many higher mass charmonium states and allow for searching for new $X, Y, Z$ particles.

\section{References}

[1] J. E. Augustin et al., Phys. Rev. Lett. 33, 1406 (1974).

[2] J. J. Aubert et al., Phys. Rev. Lett. 33, 1404 (1974).

[3] S. L. Glashow, J. Iliopoulos, L. Maiani, Phys. Rev. D 2, 1285 (1970).

[4] M. L. Perl et al., Phys. Rev. Lett. 35, 1489 (1975). 
[5] F. A. Harris (for the BES collaboration), Int. J. Mod. Phys. A 24, 377 (2008); also M. Ablikim et al. (BESIII Collab.), Nucl Instrum. and Meth A 614, 345 (2010).

[6] J. L. Rosner et al. (CLEO Collab.), Phys. Rev. Lett. 95, 102003 (2005).

[7] S. Dobbs et al. (CLEO Collab.), Phys. Rev. Lett. 101, 182003 (2008).

[8] M. Ablikim et al. (BESIII Collab.), Phys. Rev. Lett. 104, 132002 (2010).

[9] Y. P. Kuang, Phys. Rev. D 65, 094024 (2002).

[10] S. Godfrey and J. L. Rosner, Phys. Rev. D 66, 014012 (2002).

[11] C. Amsler and F. E. Close, Phys. Rev. D 53, 295 (1996).

[12] J. Bolz et al., Eur. Phys. J. C2, 705 (1998).

[13] M. Ablikim et al. (BESIII Collab.), Phys. Rev. D 81, 052005 (2010).

[14] D. M. Asner et al. (CLEO Collab.), Phys. Rev. D 79, 072007 (2009).

[15] K. Nakamura et al., J. Physics G 37, 075021 (2010).

[16] M. Ablikim et al. (BESIII Collab.), Phys. Rev. D 83, 012006 (2011).

[17] J. V. Bennett et al. (CLEO Collab.), Phys. Rev. Lett. 101, 151801 (2008). Values for $f_{t}$ are calculated from CLEO-c's $A_{ \pm} / A_{0}$.

[18] Y. J. Gao et al., hep-ph/0701009.

[19] D. Y. Chen et al., Eur. Phys. J C 70, 177 (2010).

[20] M. Ablikim et al. (BES Collab.), submitted to Phys. Rev. D, arXiv:1103.5564 (2011).

[21] M. Ablikim et al. (BES Collab.), Phys. Rev. Lett. 91, 022001 (2003).

[22] E. Fermi and C. N. Yang, Phys. Rev. 76, 1739 (1949).

[23] M. Ablikim et al. (BESIII Collab.), Chinese Physics C 34, 421 (2010).

[24] J. P. Alexander et al. (CLEO Collab.), arXiv:1007.288 (2010).

[25] M. Ablikim et al. (BES Collab.), Phys. Rev. Lett. 95, 062001 (2005).

[26] G. J. Ding and M. L. Yan, Phys. Rev. D 72, 015208 (2005).

[27] M. Ablikim et al. (BES Collab.), Phys. Rev. Lett. 106, 072002 (2011).

[28] N. N. Achasov et al., Phys. Lett. 88B, 367 (1979).

[29] J. Wu et al., Phys. Rev. D 75, 114012 (2007); C. Hanhart et al., Phys. Rev. D 76, 074428 (2007).

[30] J. Wu and B. Zou, Phys. Rev. D 78, 074017 (2008).

[31] M. Ablikim et al. (BESIII Collab.), Phys. Rev. D 83, 032003 (2011).

[32] V. L. Chernyak and A. R. Zhitnitsky, Phys. Rep. 112, 173 (1984).

[33] J. G. Körner et al., Nucl. Phys. B 229, 115 (1983).

[34] G. W. Intemann, Phys. Rev. D 27, 2755 (1983).

[35] H. Fritzsch and J. D. Jackson, Phys. Rev. Lett. B 66, 365 (1977).

[36] K. T. Chao, Nucl. Phys. B 335, 101 (1990). 
[37] T. K. Pedlar et al.(CLEO Collab.), Phys. Rev. D 79, 111101 (2009).

[38] J. L. Rosner, Phys. Rev. D 79, 097301 (2009).

[39] M. Ablikim et al. (BESIII Collab.), Phys. Rev. Lett. 105, 261801 (2010).

[40] A. G. Shamov et al. (KEDR Collab.), Nucl. Phys. B (Proc. Suppl.) 181-182, 311 (2008).

[41] J. Z. Bai et al. (BES Collab.), Phys. Rev. D 53, 20 (1996).

[42] A. Pich, Acta Phys. Polon. B 38, 3449 (2007); arXiv:0711.0028 (2007). 